\title{
A general approach for robust integrated polarization rotators
}

\author{
Alonso-Ramos C., ${ }^{a}$ Halir R., ${ }^{a}$ Ortega-Moñux A., ${ }^{a}$ Cheben P., ${ }^{b}$ Vivien L., ${ }^{c}$ Molina-Fernández \\ I., ${ }^{a}$ Marris-Morini D., ${ }^{c}$ Janz S., ${ }^{b}$ Xu D.-X., ${ }^{b}$ and Schmid J. ${ }^{b}$ \\ ${ }^{a}$ Departamento Ingeniería de Comunicaciones, ETSI Telecomunicación, Universidad de \\ Málaga, 29071 Málaga, Spain \\ ${ }^{b}$ Institute for Microstructural Sciences, National Research Council Canada, 1200 Montreal \\ Rd., K1A 0R6 Ottawa, Ontario, Canada \\ ${ }^{c}$ Institut d'Electronique Fondamentale, Univ. Paris Sud, CNRS UMR8622, Bat. 220, F-91405 \\ ORSAY, France.
}

\begin{abstract}
Integrated polarization rotators suffer from very high sensitivity to fabrication errors. A polarization rotator scheme that substantially increases fabrication tolerances is proposed. In the proposed scheme, two tunable polarization phase shifters are used to connect three rotator waveguide sections. By means of properly setting the polarization phase shifters, fabrication errors are compensated and perfect polarization rotation is achieved. Analytical conditions are shown that determine the maximum deviation that can be corrected with the proposed scheme. A design example is discussed, where the thermo-optic effect is used to provide the required tunable polarization phase shifting. Calculated $40 \mathrm{~dB}$ extinction ratio is shown in presence of fabrication errors that would yield a $4 \mathrm{~dB}$ extinction ratio in the conventional approach.
\end{abstract}

Keywords: polarization diversity, integrated polarization rotator, polarization phase shift,

\section{INTRODUCTION}

High refractive index contrast platforms such as silicon-on-insulator (SOI) and indium phosphide exhibit a high integration capacity that allows the implementation of high performance devices. ${ }^{1}$ However, due to this high index contrast, there is a large mismatch in the propagation characteristics for the Transversal Electric (TE) and Transversal Magnetic (TM) modes, making the use of polarization diversity schemes necessary. ${ }^{2}$

Polarization multiplexed based modulation schemes have emerged as a promising solution to provide data rates required by the future generation optical communication systems. Spectral efficiency is doubled in polarization multiplexing schemes, compared to traditional ones, as both TE and TM polarizations are used to send information. ${ }^{3}$ A frontend receiver for this kind of modulation comprises three different subsystems: polarization diversity management, phase diversity management, and power detection. In many practical designs each subsystem is implemented on a different platform, that best matches its technological requirements, and different subsystems are connected through fiber interfaces. Monolithic integration of the full receiver is desired to reduce complexity and cost. Whereas monolithic integration of phase diversity and power detection subsystems has already been demonstrated, ${ }^{4}$ integration of polarization diversity (comprising polarization splitters and/or rotators) is still challenging.

Many alternatives can be found in literature to implement integrated polarization rotators. Most of them are based on the use of a rotator waveguide. These rotator waveguides can be implemented using slanted sidewalls, ${ }^{5}$ two-step dry etches, ${ }^{6,7}$ transversal slots $^{8}$ or longitudinal trenches. ${ }^{9}$ Also, approaches based on cross-polarization coupling $^{10,11}$ or modal evolution ${ }^{2}$ have been proposed. However, proposed integrated polarization rotators are very sensitive to fabrication errors, ${ }^{12}$ limiting their application in commercial devices.

Further author information: (Send correspondence to C. Alonso-Ramos)

C. Alonso-Ramos: E-mail: caar@ic.uma.es, Telephone: +34 952132853 


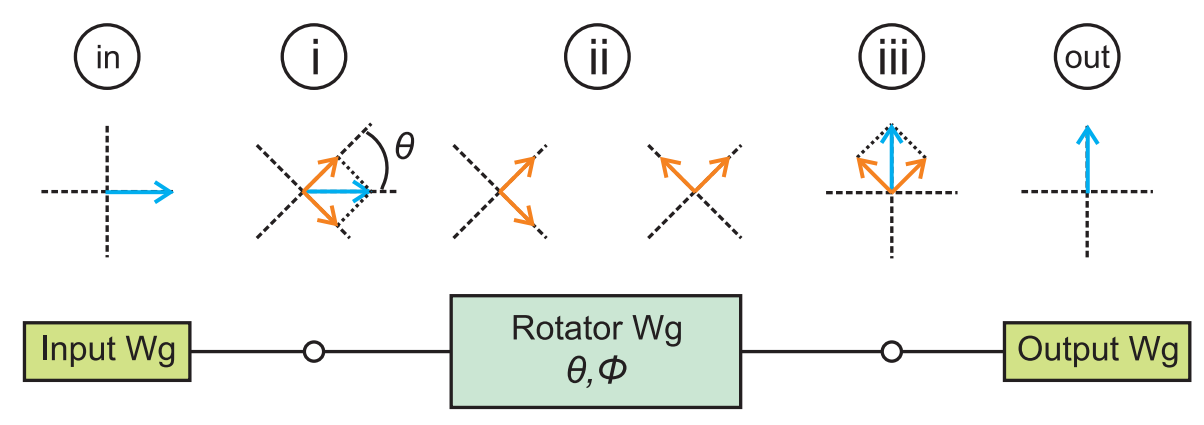

Figure 1. General approach for waveguide polarization rotator and TE to TM polarization rotation for ideal $\left(\theta=45^{\circ}\right.$, $\phi=180^{\circ}$ ) rotator waveguide.

In this work a scheme that drastically reduces the sensitivity of waveguide polarization rotators to fabrication variations is presented. The device is implemented using a rotator waveguide split in three sections joined by two polarization phase shifters. ${ }^{13}$ Assuming the behavior of the three polarization rotation sections is unknown (and uncontrolled due to fabrication errors), we show that perfect polarization rotation can be achieved by means of controlling the polarization phase shifters. Analytical conditions for the validity of the proposed approach are also given. Note that the working principle of the proposed scheme is independent of the devices used to implement the waveguide polarization rotators and polarization phase shifters, making it a general approach.

In the following section, the behavior of conventional waveguide polarization rotators is studied. In section 2 the proposed polarization rotator scheme is presented and its feasibility to provide perfect rotation is discussed. In section 3 a design example is shown, and finally some conclusions are drawn.

\section{CONVENTIONAL WAVEGUIDE POLARIZATION ROTATORS}

A waveguide polarization rotator is based on placing a rotator waveguide, that support hybrid modes that are tilted respect to the canonical TE and TM modes, between an input and output waveguides that support canonical TE and TM modes. Polarization transformation performed by the rotator waveguide can be modeled as a three steps process: i) projection from the canonical TE (TM) mode in the input waveguide onto the two hybrid modes of the rotator waveguide, ii) propagation of the two hybrid modes along the rotator waveguide, and iii) projection from the hybrid modes onto the canonical TE and TM modes of the output waveguide. This process is schematically depicted in Fig. 1 for an ideal rotator waveguide (hybrid mode tilt $\theta=45^{\circ}$ ) and TE polarized input. When the TE-polarized input mode (horizontal blue line at input) is launched into the rotator waveguide both hybrid modes (orange lines) are equally excited (step i). Due to their different propagation constants $\left(\beta_{1}, \beta_{2}\right)$, hybrid modes are phase shifted when traveling along the rotator waveguide (step ii). Relative phase shift is $\phi=\pi \cdot L_{R} / L_{\pi}$, being $L_{R}$ the rotator waveguide length, and $L_{\pi}$ the half-beat length defined as

$$
L_{\pi}=\frac{\pi}{\beta_{1}-\beta_{2}}
$$

At a distance that equals the half-beat length $\left(L_{R}=L_{\pi}\right)$ relative phase shift is $\phi=\pi$. When the $\pi$-shifted modes are projected onto the output waveguide, the TM-polarized mode (vertical blue line at output) is excited and the desired rotation is achieved (step iii).

The behavior of a waveguide polarization rotator can be modelled by the concatenation of the Jones matrix of the input-rotator projection $\left(J_{P}(\theta)\right)$, the phase shift produced in the propagation along the rotator waveguide $\left(J_{P S}(\phi)\right)$ and the rotator-output projection $\left(J_{P}(-\theta)\right)$. Being $\theta$ the hybrid modes tilt angle respect to the canonical TE (TM) modes and $\phi$ the relative phase shift induced by the rotator waveguide between its two hybrid modes, the projection and phase shift Jones matrixes can be expressed as

$$
J_{P}(\theta)=\left[\begin{array}{cc}
\cos (\theta) & \sin (\theta) \\
-\sin (\theta) & \cos (\theta)
\end{array}\right]
$$




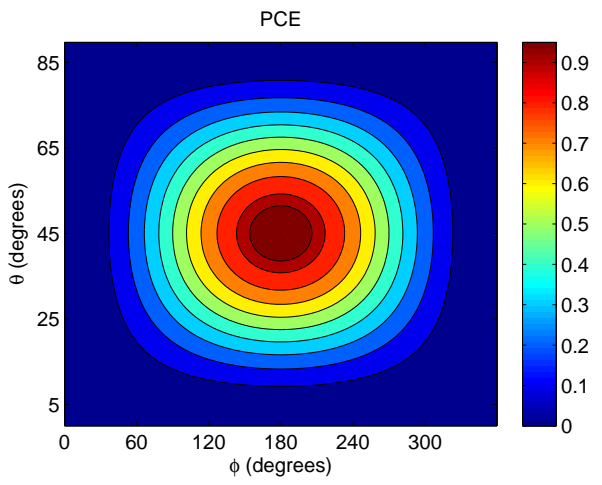

Figure 2. Calculated polarization conversion efficiency $(P C E)$ in conventional approach as a function of the rotator waveguide hybrid modes tilt angle $(\theta)$ and the differential phase shift $(\phi)$.

$$
J_{P S}(\phi)=\left[\begin{array}{cc}
e^{(j \phi)} & 0 \\
0 & 1
\end{array}\right]
$$

and the Jones matrix of the rotator waveguide $\left(J_{R}(\theta, \phi)\right)$ can be written as

$$
J_{R}(\theta, \phi)=\left[\begin{array}{cc}
e^{(j \phi)} \cos ^{2}(\theta)+\sin ^{2}(\theta) & \frac{e^{(j \phi)}-1}{2} \sin (2 \theta) \\
\frac{e^{(j \phi)}-1}{2} \sin (2 \theta) & \cos ^{2}(\theta)+e^{(j \phi)} \sin ^{2}(\theta)
\end{array}\right]
$$

being the input $\left(A_{\text {in }}\right)$ and output $\left(A_{\text {in }}\right)$ polarization states are related by

$$
A_{\text {out }}=J_{R}(\theta, \phi) \cdot A_{\text {in }}
$$

Polarization conversion efficiency $\left(P C E_{T E(T M)}\right)$ is defined as the ratio of TE (TM) injected power that is transformed into the TM (TE) polarization. Using eq. 4, $P C E_{T E(T M)}$ can be calculated as

$$
P C E_{T E(T M)}=\frac{P_{T E(T M)}}{P_{T E}+P_{T M}}=\left|J_{R_{21(12)}}(\theta, \phi)\right|^{2}=\sin ^{2}(2 \theta) \cdot \sin ^{2}(\phi / 2)
$$

where $P_{T E}, P_{T M}$ are the powers at the output of the rotator in the TE and TM polarizations components respectively, and $J_{R_{i j}}(\theta, \phi)$ is the element in row $i$ and column $j$ in the rotator waveguide Jones matrix in eq. 4. It can be seen that perfect rotation $(P C E=1)$ can only be achieved for $\theta=45^{\circ}$ and $\phi=180^{\circ}$. Device performance quickly deteriorates when rotator parameters deviate from the optimum ones. This effect is clearly seen in Fig. 2 where $P C E$ is plot as a function of $\theta$ and $\phi$. In order to have $P C E>0.95$ deviations smaller than $\Delta \phi= \pm 20^{\circ}$ for the differential phase shift and $\Delta \theta= \pm 5^{\circ}$ for the hybrid modes tilt angle should be provided. Unfortunately, fabrication errors smaller than $50-100 \mathrm{~nm}^{7,14}$ in the transversal geometry of the rotator waveguide are required to meet this condition, complicating the realization of waveguide polarization rotator with high $P C E$.

\section{PROPOSED POLARIZATION ROTATOR SCHEME}

In order to reduce waveguide polarization rotators sensitivity to fabrication tolerances, a different approach has been proposed, ${ }^{13}$ based on the use of the general scheme shown in Fig. 3. The proposed polarization rotator scheme is composed by three rotator waveguide sections $\left(\mathrm{RWS}_{i}\right)$ and two tunable polarization phase shifters $\left(\mathrm{PPS}_{i}\right)$. The behavior of each rotator waveguide section $\left(\mathrm{RWS}_{i}\right)$ is defined by the Jones matrix in eq. 4 particularized for its hybrid mode tilt angle $\left(\theta_{i}\right)$ and differential phase shift $\left(\phi_{i}\right)$. The behavior of each tunable $\mathrm{PPS}_{i}$ is defined by the Jones matrix in eq. 3 particularized for its differential phase shift $\left(\phi=\rho_{i}\right)$. As it will later be shown, this scheme provides perfect polarization rotation $(P C E=1)$ for a wide range of $\theta_{i}$ and 
(A)

(B)

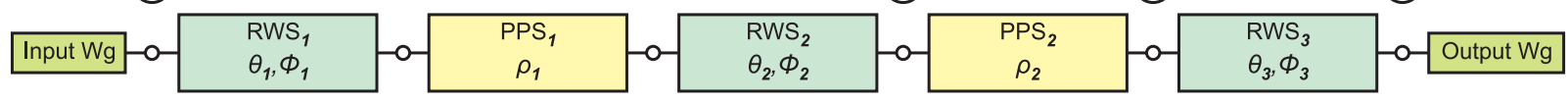

Figure 3. Proposed polarization rotator scheme composed by three rotator waveguide sections $\left(\mathrm{RWS}_{i}\right)$ and two polarization phase shifters $\left(\mathrm{PPS}_{i}\right)$.

$\phi_{i}$ by means of properly choosing the values for $\rho_{i}$. Thus, in order to provide the desired performance, $\rho_{i}$ is controllable using for example the thermo-optical or the electro-optical effects.

Conditions for perfect TE to TM polarization rotation are derived based on the concatenation of the Jones matrixes of the different elements composing the rotator scheme. Derived conditions are also valid for TM to TE polarization rotation.

Assuming an arbitrary polarization state $\left(A_{i n}\right)$ at the input of a rotator waveguide defined as

$$
A_{\text {in }}=\left[\begin{array}{l}
A_{i n}^{T E} \\
A_{\text {in }}^{T M}
\end{array}\right]=\left[\begin{array}{c}
a e^{j \rho} \\
\sqrt{1-a^{2}}
\end{array}\right]
$$

being $A_{i n}^{T E}$ and $A_{i n}^{T M}$ the amplitudes of the TE and TM components. Polarization state $\left(A_{\text {out }}\right)$ at the output of this rotator waveguide can be calculated, using eq. 5 , as

$$
A_{\text {out }}=\left[\begin{array}{c}
A_{\text {out }}^{T E} \\
A_{\text {out }}^{T M}
\end{array}\right]=\left[J_{R}(\theta, \phi)\right] \cdot\left[\begin{array}{c}
a e^{j \rho} \\
\sqrt{1-a^{2}}
\end{array}\right]
$$

and power in the TM component $\left(P_{\text {out }}^{T M}\right)$ is calculated as

$$
P_{\text {out }}^{T M}=\left|A_{\text {out }}^{T M}\right|^{2}=\left|a e^{j \rho}\left(e^{j \phi}-1\right) \sin (2 \theta) / 2+\sqrt{1-a^{2}}\left(\cos ^{2}(\theta)+e^{j \phi} \sin ^{2}(\theta)\right)\right|^{2}
$$

$P_{\text {out }}^{T M}$ can be expressed as a sinusoidal function of $\rho$ as

$$
\begin{gathered}
P_{\text {out }}^{T M}=\left[a \cdot \alpha+\sqrt{1-a^{2}} \cdot \gamma \cdot \sin (\rho+\phi / 2)\right]^{2} \\
\alpha=\sin (2 \theta) \cdot \sin (\phi / 2) \\
\gamma=\sqrt{\cos ^{4}(\theta)+\sin ^{4}(\theta)+2 \cdot \cos ^{2}(\theta) \cdot \sin ^{2}(\theta) \cdot \cos (\phi)}
\end{gathered}
$$

Note that the parameters $\alpha$ and $\gamma$ depend only on the rotator waveguide parameters $\theta$ and $\phi$, and thus are independent from the polarization state at the input. Maximum and minimum achievable $P_{\text {out }}^{T M} \operatorname{depend~on~} \theta, \phi$ and $a$ (but not on $\rho$ ) and can be calculated as

$$
\begin{aligned}
& P_{\text {out }_{\text {Max }}}^{T M}=\left[a \cdot \alpha+\sqrt{1-a^{2}} \cdot \gamma\right]^{2} \\
& P_{\text {out }_{\text {min }}}^{T M}=\left[a \cdot \alpha-\sqrt{1-a^{2}} \cdot \gamma\right]^{2}
\end{aligned}
$$

If we define $\rho^{\text {opt }}$ as the value that maximizes $P_{\text {out }}^{T M}\left(P_{\text {out }}^{T M}=P_{\text {out }}^{T M a x}\right)$, and assume we set $\rho=\rho^{\text {opt }}$, it can be demonstrated that all the power can be transferred into the TM polarization at the output of rotator $\left(P_{\text {out }_{\text {Max }}}^{T M}=1\right)$ if

$$
a=\alpha \Leftrightarrow \sqrt{1-a^{2}}=\gamma
$$


Thus, it can be concluded that for any given waveguide rotator (defined by $\theta, \phi$ ) there is an input polarization state $\left(A_{i n}^{R o t}\right)$ that provides total power transfer to the TM polarization.

$$
A_{\text {in }}=A_{\text {in }}^{\text {Rot }}=\left[\begin{array}{c}
\alpha e^{j \rho^{o p t}} \\
\gamma
\end{array}\right] \Leftrightarrow P_{\text {out }}^{T M}=1
$$

Analogous condition can be derived for total power transfer to TE polarization.

Let us consider now the proposed rotator scheme (Fig. 3), and the TE to TM polarization rotation case. In this case light at the input (point A in Fig. 3) is TE polarized $\left(P_{i n}^{T E}=1\right.$ and $\left.P_{i n}^{T M}=0\right)$ and the aim is getting TM polarized light $\left(P_{\text {out }}^{T E}=0\right.$ and $P_{\text {out }}^{T M}=1$ ) at the output (point $\mathrm{D}$ in Fig. 3). From the later discussion it is clear that, in order to provide the desired performance, polarization state at the input of the $\mathrm{RWS}_{3}$ (point $\mathrm{C}$ in Fig. 3) should meet the condition

$$
A_{C}=A_{i n_{3}}^{R o t}=\left[\begin{array}{c}
\alpha_{3} e^{j \rho^{o p t}} \\
\gamma_{3}
\end{array}\right]
$$

where $\alpha_{3}$ and $\gamma_{3}$ are calculated particularizing eq. 11 for the $\operatorname{RWS}_{3}$ characteristic parameters $\left(\theta_{3}, \phi_{3}\right)$. Required differential phase shift $\left(\rho^{o p t}\right)$ can be provided by appropriately tuning the phase shift, $\rho_{2}$, introduced by the second polarization phase shifter $\left(\mathrm{PPS}_{2}\right)$. Thus, as polarization phase shifters do not change the TE-TM power ratio, a necessary condition for perfect rotation can be defined for the power of the polarization state $\left(P_{B}\right)$ at the input of $\mathrm{PPS}_{2}$ (point B in Fig. 3) as

$$
P_{B}=\left|A_{i n_{3}}^{R o t}\right|^{2}=\left[\begin{array}{c}
P_{B}^{T E} \\
P_{B}^{T} M
\end{array}\right]=\left[\begin{array}{l}
\left|\alpha_{3}\right|^{2} \\
\left|\gamma_{3}\right|^{2}
\end{array}\right]
$$

Following the same approach used to derive eq. 10, power in TM component in point $\mathrm{B}\left(P_{B}^{T M}\right)$ for input TE polarized light $\left(P_{i n}^{T E}=1\right.$ and $\left.P_{i n}^{T M}=0\right)$ can be expressed as a sinusoidal function of $\rho_{1}$ (differential polarization phase shift introduced by $\mathrm{PPS}_{1}$ ) whose maximum and minimum values can be expressed as

$$
\begin{aligned}
& P_{B_{M a x}}^{T M}=\left[\alpha_{2} \gamma_{1}+\alpha_{1} \gamma_{2}\right]^{2} \\
& P_{B_{\text {min }}}^{T M}=\left[\alpha_{2} \gamma_{1}-\alpha_{1} \gamma_{2}\right]^{2}
\end{aligned}
$$

where $\alpha_{i}$ and $\gamma_{i}$ are calculated particularizing eq. 11 for the characteristic parameters $\left(\theta_{i}, \phi_{i}\right)$ of the corresponding $\operatorname{RWS}_{i}$. Always that the interval $\left[P_{B_{M a x}}^{T M}, P_{B_{\text {min }}}^{T M}\right]$ contains the value $\left|\gamma_{3}\right|^{2}$, there is a $\rho_{1}$ that ensures that condition in eq. 16 is met.

Thus it can be concluded that, for the proposed polarization rotator scheme, perfect TE to TM polarization rotation can be achieved, tuning the differential phase shifts $\left(\rho_{1}, \rho_{2}\right)$ induced by $\mathrm{PPS}_{1}$ and $\mathrm{PPS}_{2}$, always that $\mathrm{RWS}_{i}$ meet the condition

$$
\left[\alpha_{2} \cdot \gamma_{1}-\alpha_{1} \cdot \gamma_{2}\right]^{2}<\left|\gamma_{3}\right|^{2}<\left[\alpha_{2} \cdot \gamma_{1}+\alpha_{1} \cdot \gamma_{2}\right]^{2}
$$

This condition determines feasibility of the proposed polarization rotator scheme to provide perfect rotation polarization for different deviations from the optimal parameters in the waveguide rotators.

If we assume every rotator waveguide $\mathrm{RWS}_{i}$ has the same characteristic parameters $\left(\theta_{1}=\theta_{2}=\theta_{3}=\theta\right.$ and $\left.\phi_{1}=\phi_{2}=\phi_{3}=\phi\right)$ condition in eq. 18 reduces to

$$
\sin ^{2}(2 \theta) \cdot \sin ^{2}(\phi / 2)>\frac{1}{4}
$$

Equation 19 defines a region where perfect rotation is feasible, provided the optimum $\rho_{1}$ and $\rho_{2}$ are chosen. This region is depicted in Fig. 4, showing that perfect rotation $(P C E=1)$ is feasible even for deviations of $\Delta \phi= \pm 120^{\circ}$ in the differential phase shift or $\Delta \theta= \pm 30^{\circ}$ in the hybrid modes tilt angle. This significantly relaxes the fabrication tolerances for the waveguide rotator. 


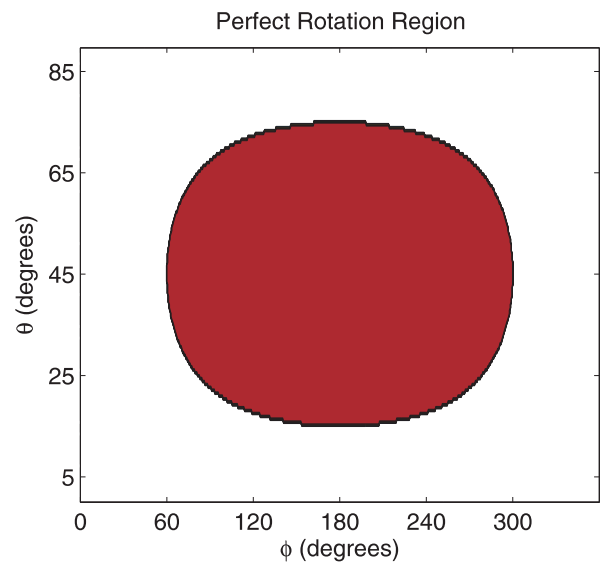

Figure 4. Region where prefect polarization rotation is feasible (eq. 19 is met).

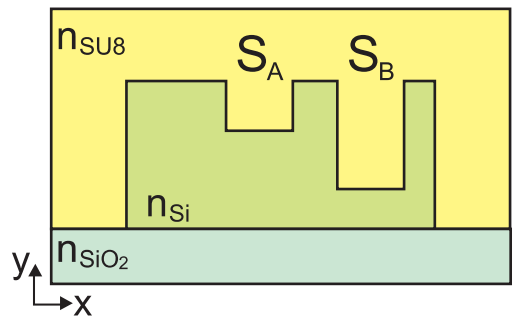

Figure 5. Transversal geometry of rotator waveguide.

\section{DESIGN EXAMPLE}

Finally we present an example of the performance improvement provided by the proposed scheme in presence of fabrication errors. We considered the waveguide rotator proposed by Velasco et al.,$^{9}$ whose transversal geometry is shown in Fig. 5. In this case, desired hybrid mode tilt angle $\left(\theta=45^{\circ}\right)$ is achieved by properly choosing the slots dimensions $\left(S_{1}, S_{2}\right.$ in Fig. 5), and desired differential phase shift $\phi=180^{\circ}$ is achieved by properly choosing waveguide rotator length $\left(L_{R}\right)$.

Extinction ratio (ER) is studied for the rotator when a $10 \%$ variation in slot dimensions is considered. At a wavelength of $\lambda=1.55 \mu \mathrm{m}$ the hybrid mode tilt angle is $\theta=25^{\circ}$, that clearly deviates form the ideal $\theta=45^{\circ}$. Figure 6 shows calculated $E R$ of the rotator as a function of $L_{R}$. The device exhibits an $E R$ of around $4 \mathrm{~dB}$, independently from the rotator waveguide length used.

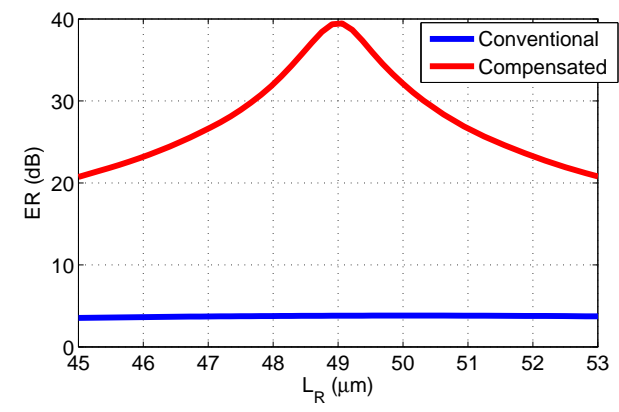

Figure 6. Calculated extiction ratio of a rotator waveguide with a large fabrication error (hybrid mode tilt angle $\theta=25^{\circ}$ ) as a function of rotator waveguide length $\left(L_{R}\right)$ for the conventional approach (blue line) and the proposed rotator scheme (red line). 
The same waveguide rotator, with the same $10 \%$ fabrication error, is used to implement the rotator waveguide sections $\mathrm{RWS}_{i}$ in our rotator scheme. Tunable polarization phase shifters $\mathrm{PPS}_{i}$ are implemented using the thermo-optical effect with waveguide heaters. ${ }^{15}$ Waveguide length of the $\mathrm{PPS}_{i}$ is set to $700 \mu m$ so $360^{\circ}$ phase shifts can be induced with temperature changes lower than $\Delta T<40^{\circ} .^{7}$ Thus device length is around $1.5 \mathrm{~mm}$. Note that device length can be reduced if higher temperature changes are considered. Calculated $E R$ of the complete rotator scheme as a function of $L_{R}$ is shown in Fig. 6. A remarkable 35dB improvement in extinction ratio is achieved.

\section{CONCLUSIONS}

A polarization rotator scheme that substantially reduces sensitivity to fabrication errors is proposed. This scheme is composed by three rotator waveguide sections and two tunable polarization phase shifters. Deviations from the optimal rotation waveguide parameters are compensated by properly adjusting the polarization phase shifters, providing perfect polarization rotation. Analytical conditions are derived to determine perfect rotation feasibility for a given combination of rotator waveguide parameters. A calculated $35 \mathrm{~dB}$ extinction ratio improvement, compared to the conventional approach in presence of fabrication errors is achieved.

The authors want to aknowledge Universidad de Málaga, Campus de Excelencia Internacional Andalucía Tech for their support.

\section{REFERENCES}

[1] Halir, R., Roelkens, G., Ortega-Moñux, A., and Wangüemert-Pérez, J., "High-performance $90^{\circ}$ hybrid based on a silicon-on-insulator multimode interference coupler," Opt. Lett. 36(2), 178-180 (2011).

[2] Barwicz, T., Watts, M. R., Popović, M. A., Rakich, P. T., Socci, L., Kärtner, F. X., Ippen, E. P., and Smith, H. I., "Polarization-transparent microphotonic devices in the strong confinement limit," Nat. Photonics 1(1), 57-60 (2006).

[3] Ip, E., Lau, A. P. T., Barros, D. J., and Kahn, J. M., "Coherent detection in optical fiber systems," Opt. Express 16(2), 753-791 (2008).

[4] Kunkel, R., Bach, H.-G., Hoffmann, D., Weinert, C., Molina-Fernández, I., and Halir, R., "First monolithic inp-based 90-hybrid oeic comprising balanced detectors for 100ge coherent frontends," in [Indium Phosphide G Related Materials, 2009. IPRM'09. IEEE International Conference on], 167-170, IEEE (2009).

[5] Deng, H., Yevick, D. O., Brooks, C., and Jessop, P. E., "Design rules for slanted-angle polarization rotators," J. Lightwave Technol. 23(1), 432 (2005).

[6] Vermeulen, D., Selvaraja, S., Verheyen, P., Bogaerts, W., Van Thourhout, D., and Roelkens, G., "High efficiency broadband polarization rotator on silicon-on-insulator," in [Group IV Photonics (GFP), 2010 7th IEEE International Conference on], 42-44, IEEE (2010).

[7] Alonso-Ramos, C., Romero-García, S., Ortega-Moñux, A., Molina-Fernández, I., Zhang, R., Bach, H., and Schell, M., "Polarization rotator for inp rib waveguide," Opt. Lett. 37(3), 335-337 (2012).

[8] Kotlyar, M. V., Bolla, L., Midrio, M., O'Faolain, L., and Krauss, T. F., "Compact polarization converter in inp-based material," Opt. Express 13(13), 5040-5045 (2005).

[9] Velasco, A. V., Calvo, M. L., Cheben, P., Ortega-Moñux, A., Schmid, J. H., Alonso-Ramos, C., Fernandez, Í. M., Lapointe, J., Vachon, M., Janz, S., et al., "Ultracompact polarization converter with a dual subwavelength trench built in a silicon-on-insulator waveguide," Opt. Lett. 37(3), 365-367 (2012).

[10] Dai, D. and Bowers, J. E., "Novel concept for ultracompact polarization splitter-rotator based on silicon nanowires," Opt. Express 19(11), 10940-10949 (2011).

[11] Liu, L., Ding, Y., Yvind, K., and Hvam, J. M., "Efficient and compact te-tm polarization converter built on silicon-on-insulator platform with a simple fabrication process," Opt. Lett. 36(7), 1059-1061 (2011).

[12] Deng, H., Yevick, D. O., Brooks, C., and Jessop, P. E., "Fabrication tolerance of asymmetric silicon-oninsulator polarization rotators," J. Opt. Soc. Am. A 23(7), 1741-1745 (2006).

[13] Alonso-Ramos, C., Halir, R., Ortega-Moñux, A., Cheben, P., Vivien, L., Molina-Fernández, Í., MarrisMorini, D., Janz, S., Xu, D.-X., and Schmid, J., "Highly tolerant tunable waveguide polarization rotator scheme," Opt. Lett. 37(17), 3534-3536 (2012). 
[14] Van der Tol, J. J., Felicetti, M., and Smit, M. K., "Increasing tolerance in passive integrated optical polarization converters," J. Lightwave Technol. 30(17), 2884-2889 (2012).

[15] Kim, J.-W., Park, S.-H., Chu, W.-S., and Oh, M.-C., "Integrated-optic polarization controllers incorporating polymer waveguide birefringence modulators," Opt. Express 20(11), 12443-12448 (2012). 